\title{
Optimal-Power Superposition Modulation for Scalable Video Broadcasting
}

\author{
Yanqing Zhang, Jiankang Zhang, Chao Xu, Mohammed El-Hajjar, and Lajos Hanzo,
}

\begin{abstract}
To mitigate the burden of the tele-traffic imposed by video streaming, Scalable Video Coding (SVC) is invoked for mapping the video clips to multiple layers, which allows us to improve the coverage quality. Although numerous nonorthogonal techniques have been conceived in the literature for maximizing the theoretical capacity relying on the idealized simplifying assumption of perfect channel coding. There is a paucity of practical finite-delay channel-coded solutions capable of mitigating the avalanche-like error proliferation routinely encountered in the face of hostile channels. Against this background, we propose SVC based Superposition Coding (SC) assisted video broadcasting, which curbs the error propagation introduced both by the inter-layer dependency and the Successive Interference Cancellation (SIC) required by the superimposed signal. Specifically, we formulate an Objective Function (OF) based on the average video quality across the Base Station's (BS) coverage area and then determine the optimal power scaling coefficients of each video layer using a bespoke Evolutionary Algorithm (EA). Our solution strikes a compelling compromise between the best possible video service provided for the cellcentre and the cell-edge users. Explicitly, our simulation results show that the optimal-power SC system guarantees a better compromise than its Time Division Multiplexing (TDM) and conventional QAM assisted counterparts, despite its reduced receiver complexity.
\end{abstract}

Index Terms-SVC, layered video broadcasting, global optimization, power optimization.

\section{INTRODUCTION}

The high data rate requirement of flawless lip-synchronized video transmission pushes the limits of the existing wireless networks. Hence, high-compression techniques have been developed, such as the H.265/High Efficiency Video Coding (HEVC) [1] scheme. In order to satisfy the ever-increasing data rate requirements of the users, substantial research efforts have been investigated in improving the bandwidth efficiency without degrading the user's quality of experience. In wireless systems, the users requiring the same content may be served within the same bandwidth.

Additionally, Cover proved in [2] that in the broadcast channel a high-rate and a low-rate information stream may be readily superimposed without any detrimental effect rather than using time-sharing. This philosophy was then generalized by Bergmans [3] to Binary Symmetric Channel (BSC), which is now generally known as Superposition Coding (SC).

M. El-Hajjar would like to acknowledge the financial support of the Royal academy of Engineering Industrial Fellow Grant.

L. Hanzo would like to acknowledge the financial support of the Engineering and Physical Sciences Research Council projects EP/Noo4558/1, EP/PO34284/1, COALESCE, of the Royal Society's Global Challenges Research Fund Grant as well as of the European Research Council's Advanced Fellow Grant QuantCom.
Furthermore, Scalable Video Coding (SVC) has attracted substantial attention as a potential solution for improving the bandwidth efficiency by mapping the video clip to multiple layers [4], including a Base Layer (BL) and several Enhancement Layers (EL)s, which are simulcast to a group of users. This compelling SVC concept has been amalgamated with the SC technique for mapping multiple video layers to a single stream [5]. Specifically, the authors of [6] quantified the performance improvement achieved by the SC aided Scalability extension of HEVC (SHVC) in four different service scenarios, while taking into account various BL and EL configurations at different data rates. Additionally, the authors of [7] formulated the SC assisted optimal beamforming design as a non-convex power minimization problem, where a greedy algorithm was developed for finding a near-optimal solution for maximizing the total utility.

Although numerous optimal power allocation schemes were conceived for video streaming, they mainly focused on maximizing the theoretical Shannon capacity [7]. By contrast, there is a paucity of contributions on finding the optimal power sharing for SC system, especially when considering the practical error propagation and interference effects. Against this background, we design an optimal-power SC-assisted layered video broadcast system, which mitigates the error proliferation imposed by realistic finite-delay channel coding. Explicitly, we mathematically model the relationship between the power sharing coefficients assigned to different video layers and the resultant Packet Loss Ratio (PLR) of different modulation modes, when Successive Interference Cancellation (SIC) aided reception is employed. Explicitly, the power sharing coefficients of the video layers are optimized by our bespoke bio-inspired global optimization algorithm [8]. The simulation results show that the proposed optimal-power SC scheme provides the best $\mathrm{BL}$ service coverage, despite its lowest detection complexity.

The rest of the paper is organized as follows. Section II depicts the system model, while the power optimization is illustrated in Section III. We compare the video performance versus the demodulation complexity of different schemes in Section IV, and finally we conclude in Section V.

\section{System Model}

In this section we highlight our layered video broadcasting scheme of Fig. 1, while the optimization block of Fig. 1 will be detailed in Section III. 


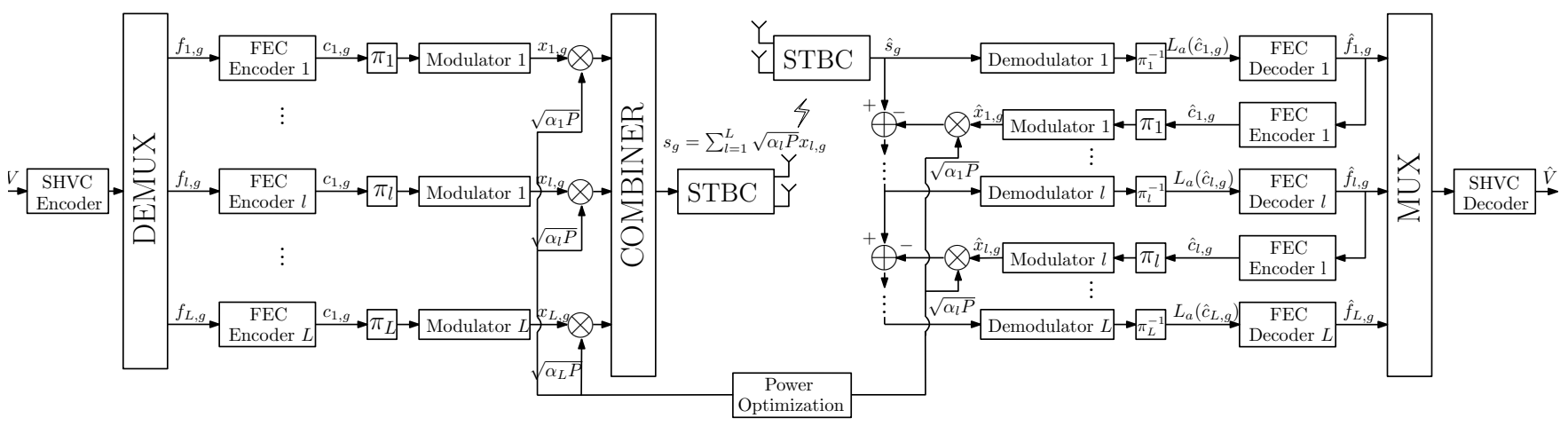

Fig. 1. The transmitter and receiver model.

\section{A. Transmitter Model}

As shown in Fig. 1, the captured video sequence $V$ is encoded using the SHVC encoder that compresses the video clips into $L$ layers $\left\{f_{l, g}\right\}_{l=1}^{L}$, where $f_{l, g}$ represents the $g$-th video frame of the $l$-th layer. Then, these bit streams are first encoded by their corresponding Forward Error Correction (FEC) encoders, which generate the encoded bit streams of $\left\{c_{l, g}\right\}_{l=1}^{L}$. The encoded bit streams are then fed into $L$ interleavers $\left\{\pi_{l}\right\}_{l=1}^{L}$, as shown in Fig. 1, before mapping them to their associated modulators, where $L$ modulated signals $\left\{x_{l, g}\right\}_{l=1}^{L}$ are generated. Afterwards, the modulated symbols are scaled by the optimal power coefficients of $\left\{\alpha_{l}\right\}_{l=1}^{L}$, which are also known at the receiver. Finally, these signals are superimposed according to $s_{g}=\sum_{l=1}^{L} \sqrt{\alpha_{l} P} x_{l, g}$, before Space-Time Block Coding (STBC) for transmission.

\section{B. Receiver Model}

The receiver model is shown in Fig. 1, where the SIC technique is invoked for progressively peeling off the superimposed signals of different layers. The STBC decoded signal $\hat{s}_{g}$ is first demodulated by Demodulator 1 of Fig. 1, while the signals of the other layers are treated as interference. Following deinterleaving, the deinterleaved Log-Likelihood Ratio (LLR) $L_{a}\left(\hat{c}_{1, g}\right)$ are then fed into the FEC Decoder 1, generating the decoded version of the BL stream, $\hat{f}_{1, g}$, which can be fed into the video decoder for reconstructing the BL. In order to recover the ELs, we have to remove the interference imposed by the $\mathrm{BL}$ and hence a re-encoding process is invoked for reconstructing $x_{1, g}$. Hence we have regenerated the modulated signal $\hat{x}_{1, g}$, as shown in Fig. 1, where $\hat{x}_{1, g}$ is expected to be identical to $x_{1, g}$ if $\hat{f}_{1, g}$ is successfully decoded. Following power-scaling by $\sqrt{\alpha_{1} P}$, the BL signal can be removed from the superimposed signal, and then the receiver proceeds with decoding the first EL, which is similar to that of decoding the BL by treating the bits of $[l+1, L]$ layers as interference. The SIC procedure continues until the highest EL $\hat{f}_{L, g}$ is decoded. The decoded video layers $\left\{\hat{f}_{l, g}\right\}_{l=1}^{L}$ are then assembled into the SHVC bit stream by the MUX block of Fig. 1 to reconstruct the decoded video $\hat{V}$.

\section{POWER OPTIMIZATION}

The modulated and scaled signals are superimposed before their transmission, as shown in Fig. 1, which are uniquely and unambiguously distinguished by the power assigned. This section is focused on the "Power Optimization" block of Fig. 1, which aims for finding the optimal power allocation scheme for the sake of maximizing the average video quality across the service coverage area of the Base Station (BS).

\section{A. Problem Formulation}

The problem is formulated for a layered video based open-loop broadcast system operating without requiring any knowledge of the Channel State Information (CSI). In this section, we design an Objetive Function (OF) that empirically characterizes the relationship between the power assigned to each layer and the average video quality across the BS's coverage area. Having a total transmit power of $P$, the average received video quality across a Group of Pictures $(\mathrm{GoP})$ period of $G$ can be modelled as:

$$
\begin{array}{ll}
\max & \mathbb{E}[Q(P, \boldsymbol{\alpha})], \\
\text { s.t. : } & \sum_{l=1}^{L} \alpha_{i} \leq 1,
\end{array}
$$

where (2) represents the constraint on the power consumption, where $\boldsymbol{\alpha}=\left\{\alpha_{1}, \cdots, \alpha_{l}, \cdots, \alpha_{L}\right\}$ indicates the power scaling coefficient set of all the layers. Assuming that the users are uniformly distributed within the maximum cell radius of $S$ covered by the BS, the probability density function of their distances $s$ from the BS can be expressed as $f_{s}(s)=2 s / S^{2}$ [9]. The average video quality $\mathbb{E}[Q(P, \boldsymbol{\alpha})]$ can hence be formulated as:

$$
\mathbb{E}[Q(P, \boldsymbol{\alpha})]=\int_{0}^{S} f_{s}(s) Q(P, \boldsymbol{\alpha}, s) \mathrm{d} s,
$$

where $Q(P, \boldsymbol{\alpha}, s)$ is denoted as the video quality at the arbitrary distance $s \in[0, S]$ away from the BS. Additionally, we model the video quality experienced by taking into account the video frame length and its associated decodability, which is expressed by:

$$
Q(P, \boldsymbol{\alpha}, s)=\sum_{l=1}^{L} \sum_{g=1}^{G} \Delta q_{l, g} \Psi_{l, g}(P, \boldsymbol{\alpha}, s),
$$

where $\Delta q_{l, g}$ represents the video quality improvement experienced by the $g$-th frame upon receiving the $l$-th layer, which can be expressed as:

$$
\Delta q_{l, g}= \begin{cases}q_{l, g}, & l=1, \\ q_{l, g}-q_{l-1, g}, & 1<l \leq L .\end{cases}
$$


Furthermore, $\Psi_{l, g}(P, \boldsymbol{\alpha}, s)$ represents the equivalent Successful Decoding Probability (SDP) of the video frame $f_{l, g}$ at distance $s$, which, in addition to considering its own SDP $\psi_{l, g}$, takes into account the decodability of all its dependent frames, namely $\left\{\psi_{1,1}, \ldots, \psi_{m, n}, \ldots, \psi_{l, g}\right\}$. Explicitly, the SDP of $\Psi_{l, g}(P, \boldsymbol{\alpha}, s)$ can be expressed as:

$$
\Psi_{l, g}(P, \boldsymbol{\alpha}, s)=\prod_{m=1}^{l} \prod_{n=1}^{g} \psi_{m, n}(P, \boldsymbol{\alpha}, s) .
$$

where $\psi_{m, n}(P, \boldsymbol{\alpha}, s)$ denotes the SDP of the frame $f_{m, n}$ at distance $s$, which will be discussed in the next section.

\section{B. Successful Video Frame Decoding Probability}

In this subsection, we elaborate on modelling the SDP of the variable-length video frame for different modulation modes. It has been shown in [10] that the video Frame Error Rate (FER) imposed by the fading channel is independent of the packet length when the distribution of errors may be considered to be uniform. Conditioned on the power constraint $P$ and on the power allocation coefficient set $\boldsymbol{\alpha}$, for the $l$-th video layer, the PLR of a packet containing $\lambda$ bits at a specific distance of $s$ is $p_{l}(P, \boldsymbol{\alpha}, s)$. Then the SDP of video frame $f_{l, g}$ having an arbitrary bit length of $\left|f_{l, g}\right|$ can be expressed as:

$$
\psi_{l, g}(P, \boldsymbol{\alpha}, s)=\left[1-p_{l}(P, \boldsymbol{\alpha}, s)\right]^{\frac{\left|f_{l, g}\right|}{\lambda}} .
$$

As shown in Fig. 1 the signals of all the layers are superimposed before transmission and $p_{l}(P, \boldsymbol{\alpha}, s)$ can usually be decomposed into: 1$)$ the errors propagated from its dependent layers and, 2) its own errors. The authors of [11] demonstrated that in Turbo Coding (TC) assisted SC broadcasting systems the PLR of the EL can be approximated by the sum of the PLR of the individual ELs assuming perfect SIC plus the PLR of its dependent layers. Let us denote the PLR of the $l$-th layer by $\tilde{p}_{l}(P, \boldsymbol{\alpha}, s)$, when its dependent layers are assumed to be perfectly decoded and hence no error propagation from the lower layers is considered. The PLR of $p_{l}(P, \boldsymbol{\alpha}, s)$ can thus be modelled as:

$$
p_{l}(P, \boldsymbol{\alpha}, s) \approx \begin{cases}\tilde{p}_{1}(P, \boldsymbol{\alpha}, s), & l=1, \\ \min \left(\sum_{m=1}^{l} \tilde{p}_{m}(P, \boldsymbol{\alpha}, s), 1\right), & 1<l \leq L,\end{cases}
$$

where $\tilde{p}_{l}(P, \boldsymbol{\alpha}, s)$ is jointly determined by the received Signalto-Noise Ratio (SNR) of the $l$-th signal $\gamma_{l}(s)$ and the received SNR of the other superimposed signals $\kappa_{l}(s)$, which are respectively given by:

$$
\begin{aligned}
& \gamma_{l}(s)=10 \log _{10} P \alpha_{l}-\beta(s)-N, \\
& \kappa_{l}(s)=10 \log _{10} P \sum_{m=l+1}^{L} \alpha_{m}-\beta(s)-N,
\end{aligned}
$$

where $N$ represents the Gaussian noise power on a deciBel (dB) scale. Moreover, $\beta(s)$ represents the expected Path Loss (PL) at $s$, which is given by:

$$
\beta(s)=\bar{\beta}(s)+\chi,
$$

where $\bar{\beta}(s)$ is the PL, while $\chi$ represents the slow fading margin. More explicitly, having a standard deviation of shadow fading $\sigma$, in order to compensate $90 \%$ shadowing attenuation, $\chi$ is set to approximately $1.3 \sigma$ [12].

Furthermore, for the sake of modelling the PLR under various SNRs of $\gamma$ and $\kappa$, we first pre-record the PLR with associated parameters for each layer in a LookUp Table (LUT) using Monte Carlo simulations. More particularly, a pair of LUTs are established, namely $h_{L}[\gamma]$ and $\left\{h_{l}[\gamma, \kappa]\right\}_{l=1}^{L-1}$, where the former is used for characterizing the PLR of the highestindex EL $l_{L}$ having no additional interference, while the latter is employed for that of the remaining layers. Then, we may find $\tilde{p}_{l}(P, \boldsymbol{\alpha}, s)$ by searching through the LUT, as follows:

$$
\tilde{p}_{l}(P, \boldsymbol{\alpha}, s)= \begin{cases}h_{l}[\gamma, \kappa], & 1 \leq l<L, \\ h_{L}[\gamma], & l=L,\end{cases}
$$

which can be readily represented by mathematical models [13]. Assisted by (7)-(12), the SDP of the video frame $f_{l, g}$ hence can be expressed as:

$$
\begin{aligned}
& \psi_{l, g}(P, \boldsymbol{\alpha}, s)= \\
& \left\{\begin{array}{cl}
{\left[1-\min \left(\sum_{\substack{m=1 \\
L-1}}^{l} h_{m}\left[\gamma_{m}(s), \kappa_{m}(s)\right], 1\right)\right]^{\frac{\left|f_{l, g}\right|}{\lambda}},} & 1 \leq l<L, \\
{\left[1-\min \left(\sum_{m=1} h_{m}\left[\gamma_{m}(s), \kappa_{m}(s)\right]\right.\right.} & \\
\left.\left.+h_{L}\left[\gamma_{L}(s)\right], 1\right)\right]^{\frac{\left|f_{l, g}\right|}{\lambda}}, & l=L .
\end{array}\right.
\end{aligned}
$$

\section{Power Optimization}

Armed with the SDP model derived in Section III-B, we can rewrite the received video quality function of (1) in discrete form as the summation of the video quality at various distances, as follows:

$$
\begin{aligned}
\mathbb{E}[Q(P, \boldsymbol{\alpha})] & =\frac{2}{S^{2} G} \sum_{s=0}^{S} \sum_{l=1}^{L} \sum_{g=1}^{G} \Delta q_{l, g} \Psi_{l, g}(P, \boldsymbol{\alpha}, s) \\
& =\frac{2}{S^{2} G} \sum_{s=0}^{S} \sum_{l=1}^{L} \sum_{g=1}^{G} \Delta q_{l, g} \prod_{m=1}^{l} \prod_{n=1}^{g} \psi_{m, n}(P, \boldsymbol{\alpha}, s),
\end{aligned}
$$

where $\psi_{m, n}(P, \boldsymbol{\alpha}, s)$ is derived in (13). The optimal power scaling coefficient set can then be found by finding the solution of the problem:

$$
\{\boldsymbol{\alpha}\}_{o p t}=\underset{\substack{\boldsymbol{\alpha}=\left\{\alpha_{l}\right\}_{l=1}^{L} \in[0,1] \\ \sum_{l=1}^{L} \alpha_{l}=1}}{\arg \max } \mathbb{E}[Q(P, \boldsymbol{\alpha})] .
$$

Since the values of the power scaling coefficients are continuous, using an exhaustive search becomes infeasible. Instead, we employ Evolutionary Algorithm (EA) assisted optimization for finding the optimal power sharing coefficients, as described in Algorithm 1, which can be readily extended to arbitrary optimization algorithms [14]. In the mutation subsection of Algorithm 1, $\hat{\boldsymbol{\alpha}}_{i, p_{s}, \text { best }}$ is randomly selected from the population archive, while $c_{1}$ and $c_{2}$ constitute a pair of random integers fetched from the set $\left\{1,2, \cdots, p_{s}-1, p_{s}+1, \cdots, P_{s}\right\}$. Furthermore, $\mu_{C_{r}}$ and $\mu_{\lambda}$ in the adaptivity subsection of 
Algorithm 1 are adaptively updated using the arithmetic-mean $\overline{S_{C_{r}}^{A}}$ and Lehmer-mean $\overline{S_{\lambda}^{L}}$ operators [15], respectively.

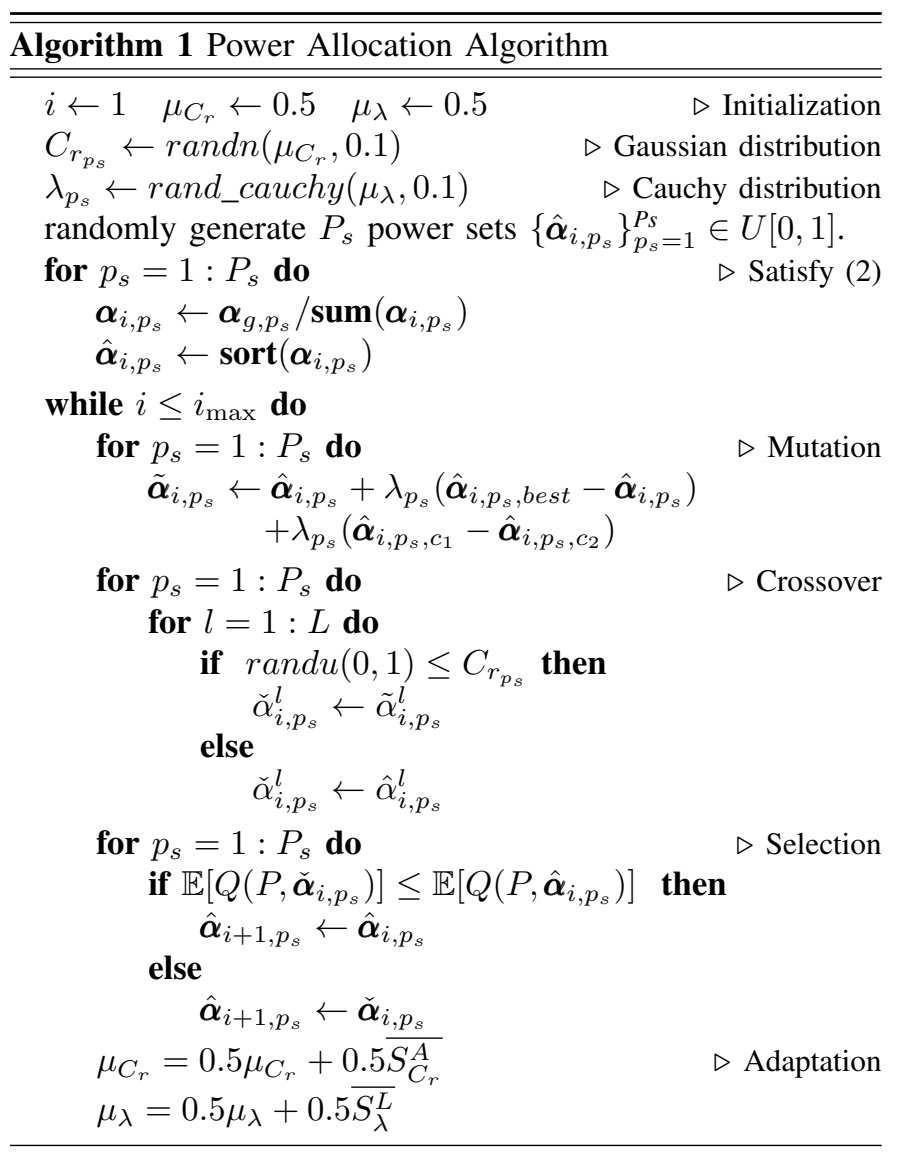

\section{Simulation Results}

In this section, we characterize the proposed optimal power seeking assisted SC-SHVC video streaming. To benchmark our system, we use both the Time Division Multiplexing (TDM) and the conventional QAM assisted systems. To elaborate, both the proposed and the TDM assisted systems rely on the SVC scheme, hence the latter is termed as TDM-SHVC. By contrast, the single-layer coded video bits are transmitted by the conventional QAM scheme, which is represented by the acronym QAM-HEVC.

A 32-frame Basketball video clip of 4:2:0 YUV format containing $1280 \times 720$ pixels is used in our simulations. In the SHVC scenario, the video clip is encoded by the SHVC scheme into three video layers having bitrates of $500 \mathrm{kbps}, 1 \mathrm{Mbps}$ and $1.5 \mathrm{Mbps}$, respectively, resulting in Peak SNR (PSNR) values of $32.3 \mathrm{~dB}, 36.4 \mathrm{~dB}$ and $38.3 \mathrm{~dB}$, respectively. In the single-layer HEVC scenario, the video clip is compressed into a $3 \mathrm{Mbps}$ bit stream having a PSNR of $40.5 \mathrm{~dB}$, hence exhibiting a marginally better PSNR than its SHVC counterpart due to the coding efficiency penalty of the scalable scheme. This can be mitigated by employing the optimal scalable video configuration of [4]. Furthermore, the GoP is set to 8 in both cases, where the bidirectional predictive frames are deactivated, since they are prone to propagating inter-frame video distortions.
Furthermore, we employ near-capacity turbo codes having a pair of identical Recursive Systematic Convolutional (RSC) components, each having the generator polynomials of [7 5]. The resultant $\frac{1}{3}$-rate code is then punctured using the

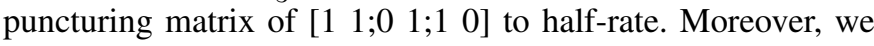
assume that a total transmit power of $40 \mathrm{dBm}$ is transmitted by a $2 \times 1 \mathrm{STBC}$ scheme over a $1 \mathrm{MHz}$-bandwidth wireless channel using a $2 \mathrm{GHz}$ carrier for broadcasting the video signals over a maximum coverage radius of $1000 \mathrm{~m}$. The PL is represented by the COST Hata model, where the BS and User Equipments (UE) heights are set to $50 \mathrm{~m}$ and $1.5 \mathrm{~m}$. The shadow fading is modelled by the classic log-normal distribution having a standard deviation of $8 \mathrm{~dB}$. Additionally, based on our empirical experiment, a population size of $P_{s}=10$ and a maximum number of $i_{\max }=30$ iterations is sufficient for the convergence of the EA. Moreover, in the proposed SC-SHVC scheme of Fig. 1, the modulation modes used for conveying the bits of the three layers are set to BPSK, 4QAM and 8PSK, respectively. Furthermore, to provide the same capacity, the TDM-SHVC scheme employs 8PSK, 64QAM and 512QAM for conveying the bits of the three layers, while the QAM-HEVC scheme activates 64QAM. Finally, the simulations are repeated 100 times.

\section{A. Quality of Experience Analysis}

The PSNR performance versus distance is depicted in Fig. 2, which shows that the QAM-HEVC scheme provides the best video quality, when the coverage radius is lower than $600 \mathrm{~m}$. This is because the PSNR of the individual video frames in the HEVC scheme is higher than that of its corresponding SHVC assisted counterparts. By contrast, both SHVC assisted schemes show a better received video quality for the cell-edge users. Fig. 3 compares the FER of the three schemes, where

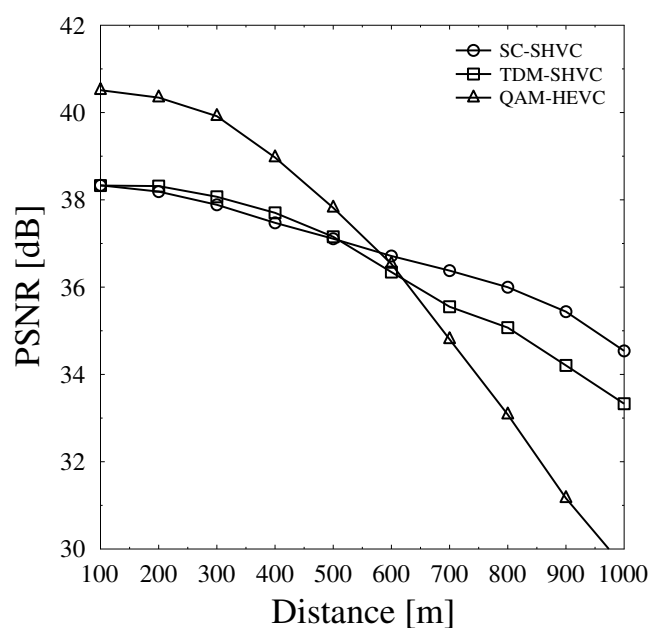

Fig. 2. PSNR $[\mathrm{dB}]$ versus the distance away from the BS between three schemes.

the BL of the two SHVC assisted arrangements is presented. It can be readily seen in Fig. 3 that the optimal power scaling assisted SC-SHVC scheme seeks the FER below 5\% upto the coverage radius of about $850 \mathrm{~m}$. By contrast, its TDM-SHVC and QAM-SHVC counterparts only cover about $700 \mathrm{~m}$ and 
$400 \mathrm{~m}$ coverage radii, when achieving a FER of $5 \%$. Observe from Fig. 2 and 3 that although the QAM-HEVC scheme provides the best image quality for the users within $600 \mathrm{~m}$ of the BS, it is no longer able to provide smooth, flawless video playback for the users further than $400 \mathrm{~m}$ from the BS. By contrast, the SHVC schemes are capable of ensuring nearperfect playback across the entire coverage area, albeit at the expense of a marginally reduced video PSNR at the cell centre compared to that of the QAM-HEVC scheme.

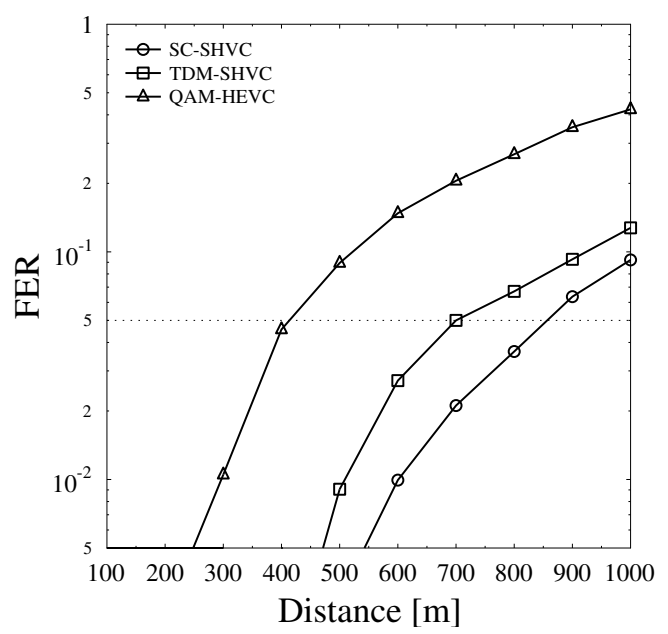

Fig. 3. FER of the BLversus the distance away from the BS between the three schemes.

\section{B. Complexity Analysis}

For the specific FEC configuration, the complexity of FEC decoding is determined by the packet length and the packet number. Due to the fact that the complexity imposed by FEC encoding and by modulation is nearly negligible compared to its decoding and demodulation counterparts, the re-encoding and the re-modulation complexity of SIC in our SC assisted scheme is omitted. Furthermore, since all the schemes in the simulation employ the same FEC configuration and hence exhibit the same FEC encoded overall rate, we assume that all the schemes share the same FEC decoding complexity. Therefore, we compare the complexity order imposed by demodulation. Table I compares the demodulation complexity of the three

Table I: Demodulation complexity comparison of the three schemes.

\begin{tabular}{|c|c|c|c|}
\hline Scheme & SC-SHVC & TDM-SHVC & QAM-HEVC \\
\hline Complexity & $\mathcal{O}(2+4+8)$ & $\mathcal{O}\left(\frac{8+64+512}{3}\right)$ & $\mathcal{O}(64)$ \\
\hline
\end{tabular}

schemes when the classic Maximum Likelihood (ML) is invoked. Specifically, in our proposed SC assisted system, the superimposed symbols have to be demodulated progressively by BPSK, 4QAM and 8PSK, yielding the complexity order of $\mathcal{O}(2+4+8)$, while its QAM-HEVC counterpart employing 64QAM yields the complexity order of $\mathcal{O}(64)$. As for the TDM-SHVC scheme, due to the fact that the three modulation modes, namely 8PSK, 64QAM and 512QAM, are activated successively, the corresponding computational complexity order becomes a mean of them, namely $\mathcal{O}\left(\frac{8+64+512}{3}\right)$. It can be seen from Table I, the SC-SHVC scheme provides a significant complexity reduction compared to the other two schemes, while the TDM-SHVC scheme exhibits the highest complexity since it requires a higher-complexity modulation mode for achieving an identical system throughput.

\section{CONCLUSION}

In this treatise, we conceived an optimal power scaling assisted SC scheme for layered video broadcasting. We first formulated an OF by carefully exploiting the inter-layer dependencies and then a novel EA algorithm was designed for finding the optimal power allocation scheme. The simulation results showed that the optimal power scaling assisted SCSHVC scheme exhibits the best video service coverage at the lowest demodulation complexity, albeit at the expense of a lower video PSNR at cell centre.

\section{REFERENCES}

[1] G. J. Sullivan, J. Ohm, W.-J. Han, and T. Wiegand, "Overview of the high efficiency video coding (HEVC) standard," IEEE Trans. Circuits Syst. Video Technol., vol. 22, no. 12, pp. 1649-1668, 2012.

[2] T. Cover, "Broadcast channels," IEEE Trans. Inf. Theory, vol. 18, no. 1 pp. 2-14, 1972.

[3] P. Bergmans, "Random coding theorem for broadcast channels with degraded components," IEEE Trans. Inf. Theory, vol. 19, no. 2, pp. $197-$ 207, 1973

[4] H. Schwarz, D. Marpe, and T. Wiegand, "Overview of the scalable video coding extension of the H.264/AVC standard," IEEE Trans. Circuits Syst. Video Technol., vol. 17, no. 9, pp. 1103-1120, 2007.

[5] L. Fay, L. Michael, D. Gómez-Barquero, N. Ammar, and M. W. Caldwell, "An overview of the ATSC 3.0 physical layer specification," IEEE Trans. Broadcast., vol. 62, no. 1, pp. 159-171, 2016.

[6] J.-y. Lee, S.-I. Park, S. Kwon, B.-M. Lim, H. M. Kim, N. Hur, A. Pesin, J.-C. Chevet, J. Llach, A. J. Stein, et al., "Efficient transmission of multiple broadcasting services using LDM and SHVC," IEEE Trans. Broadcast., vol. 64, no. 2, pp. 177-187, 2017.

[7] C. Guo, Y. Cui, D. W. K. Ng, and Z. Liu, "Multi-Quality Multicast Beamforming With Scalable Video Coding," IEEE Trans. Commun., vol. 66, no. 11, pp. 5662-5677, 2018.

[8] J. Zhang, S. Chen, X. Mu, and L. Hanzo, "Joint channel estimation and multiuser detection for SDMA/OFDM based on dual repeated weighted boosting search," IEEE Trans. Veh. Technol., vol. 60, no. 7, pp. 3265 3275, 2011.

[9] Y. I. Choi and C. G. Kang, "Scalable video coding-based MIMO broadcasting system with optimal power control," IEEE Trans. Broadcast., vol. 63, no. 2, pp. 350-360, 2016.

[10] Y. Huo, C. Hellge, T. Wiegand, and L. Hanzo, "A tutorial and review on inter-layer FEC coded layered video streaming," IEEE Commun. Surveys Tut., vol. 17, no. 2, pp. 1166-1207, 2015.

[11] H.-T. Kim, S. H. Lim, I. Lee, S. Kim, and S.-Y. Chung, "Code design for MIMO downlink with imperfect CSIT," IEEE Trans. Commun., vol. 58, no. 1, pp. 89-94, 2010.

[12] Walpole, R. E. and Myers, R. H. and Myers, S. L. and Ye, K., Probability and statistics for engineers and scientists, vol. 5. Macmillan New York, 1993.

[13] Y. Huo, C. Zhou, J. Jiang, and L. Hanzo, "Historical information aware unequal error protection of scalable HEVC/H. 265 streaming over free space optical channels," IEEE Access, vol. 4, pp. 5659-5672, 2016.

[14] X. Ge, Y. Sun, H. Gharavi, and J. Thompson, "Joint Optimization of Computation and Communication Power in Multi-User Massive MIMO Systems," Trans. Wireless Commun., vol. 17, no. 6, pp. 4051-4063, 2018.

[15] J. Zhang, S. Chen, X. Mu, and L. Hanzo, "Evolutionary-algorithmassisted joint channel estimation and turbo multiuser detection/decoding for OFDM/SDMA," IEEE Trans. Veh. Technol., vol. 63, no. 3, pp. 12041222,2014 